\title{
Perfil dos desvios posturais da coluna vertebral em adolescentes de escolas públicas do município de Juazeiro do Norte - CE
}

\author{
Profile of postural deviations of the spine in adolescents from public schools in the city of \\ Juazeiro do Norte - CE
}

José Vitorino de Souza Junior ${ }^{1}$, Rafaella Maria Monteiro Sampaio ${ }^{2}$, Jaina Bezerra de Aguiar ${ }^{3}$, Francisco José Maia Pinto ${ }^{4}$

Estudo desenvolvido em três escolas do Ensino Fundamental II e Médio do município de Juazeiro do Norte (CE), Brasil.

${ }^{1}$ Fisioterapeuta; Mestre em Saúde da Criança e do Adolescente pela Universidade Estadual do Ceará (UECE) - Fortaleza (CE), Brasil; Docente do curso de fisioterapia da Faculdade Leão Sampaio - Juazeiro do Norte (CE), Brasil.

${ }^{2}$ Nutricionista; Discente do Mestrado Acadêmico em Saúde Pública pela UECE - Fortaleza (CE), Brasil.

${ }^{3}$ Educadora Física; Discente do Mestrado Acadêmico em Saúde Pública pela UECE - Fortaleza (CE), Brasil.

${ }^{4}$ Doutor em Saúde Coletiva pelo Instituto de Medicina Social da Universidade Estadual do Rio de Janeiro (UERJ) - Rio de Janeiro (RJ), Brasil; Docente do Curso de Medicina, do Mestrado Acadêmico em Saúde Pública e do Mestrado Profissional em Saúde da Criança e do Adolescente da UECE Fortaleza (CE), Brasil.

\section{ENDEREÇO PARA CORRESPONDÊNCIA}

Rafaella Maria Monteiro Sampaio, Rua Doutor Jurandyr Nunes, 508 - Sapiranga - CEP: 60833-192 - Fortaleza (CE), Brasil - E-mail: rafaellasampaio@yahoo.com.br

\section{APRESENTAÇÃO \\ mar. 2011}

\section{ACEITO PARA PUBLICAÇÃO} out. 2011

\section{FONTE DE FINANCIAMENTO} nenhuma

\section{CONFLITO DE INTERESSE} nada a declarar

Parecer de aprovação no Comitê de Ética da UECE (processo $n^{\circ}$ 051.73.853-8)
RESUM0: Postura é definida como sendo a posição adotada pelo ser humano. Dados epidemiológicos apontam para uma alta prevalência de alterações posturais de coluna entre crianças e adolescentes. O objetivo do presente estudo foi investigar os desvios posturais da coluna vertebral em escolares $(n=670)$ de 11 a 19 anos. Utilizou-se como metodologia um simetrógrafo e uma máquina fotográfica para pesquisar a presença de desvios na coluna vertebral. Foram utilizados um nível d'água e uma régua para aferir a gibosidade, um fio de prumo e uma régua para medir os desvios laterais da coluna. Dos resultados obtidos, encontrou-se uma prevalência de $8,8 \%$ de desvios laterais e $2,4 \%$ de gibosidade, além de ser observada a presença de escoliose em portadores de assimetrias de ombros e ilíacos. Porém, não houve influência do peso, altura e índice de massa corporal (IMC) para a prevalência de escoliose. Dessa maneira, com este estudo, constatou-se elevada prevalência dos desvios posturais em escolares.

DESCRITORES: postura; escoliose; adolescente.

ABSTRACT: Posture is defined as the position taken by humans. Epidemiological data indicate a high prevalence of backbone postural changes among children and adolescents. The aim of this study was to investigate the postural deviations of the spine in schoolchildren $(n=670)$ from 11 to 19 years. The methodology used was a simetrograf and a camera for the presence of deviations in the spine. We used a water level and a ruler to measure the spinal deformity, a plumb line and a ruler to measure the lateral deviation of the spine. Of the obtained results it was found a prevalence of $8.8 \%$ of lateral deviation and $2.4 \%$ of gibbosity, besides it was observed the presence of scoliosis in patients with asymmetries in the shoulders and hipbones. However, there was no influence of weight, height and body mass index (BMI) for the prevalence of scoliosis. Thus, through this study, it was stated the high prevalence of postural deviations in schoolchildren.

KEYwords: posture; scoliosis; adolescent. 


\section{INTRODUCÃO}

Postura pode ser definida como sendo a posição corporal adotada pelo ser humano ${ }^{1}$, podendo ser influenciada por maus hábitos que podem produzir maior tensão sobre as estruturas de suporte ${ }^{2}$. As afecções do sistema músculo-esquelétiCO, particularmente as algias vertebrais, constituem problema tão sério na sociedade que equipes multidisciplinares procuram desenvolver formas para adequada avaliação da coluna vertebral ${ }^{3}$.

Em escolares, as posturas inadequadas adotadas em sala de aula e/ou em casa podem levar ao desequilíbrio na musculatura, produzindo alterações posturais ${ }^{1,4}$. Dentre as diversas alterações posturais possíveis de serem encontradas em escolares, destaca-se a escoliose ${ }^{1}$.

O termo escoliose tem origem grega e significa curvatura, sendo, portanto, definida como desvio lateral do plano frontal do corpo, associado ou não à rotação dos corpos vertebrais nos planos axial e sagital com ângulo maior que 10 $\operatorname{graus}^{2,5}$.

Dados epidemiológicos apontam para alta prevalência de alterações posturais de coluna entre crianças e adolescentes. Em relação à escoliose, a prevalência global está entre 1 e $2 \%$, e a escoliose idiopática em adolescentes é o subgrupo mais comum ${ }^{6}$.

Na grande maioria dos casos, a causa específica não é encontrada, sendo a etiologia denominada de idiopática $^{5}$. Seu desenvolvimento pode ocorrer desde a infância e agravar-se na adolescência ${ }^{2,4,7}$.

No caso da escoliose idiopática do adolescente, o momento de maior risco para a progressão da doença ocorre no período da puberdade, em que o crescimento ósseo ocorre de forma muito rápida, havendo, portanto, a necessidade de meios de diagnóstico precoce e de intervenção eficaz para evitar a progressão da doença ${ }^{5}$.

Alguns países desenvolvidos já adotam a realização sistemática de avaliações posturais durante a fase escolar para identificar e acompanhar a progressão das alterações da postura em geral e, principalmente, da postura da coluna vertebral. Desse modo, as avaliações posturais, em que os indivíduos são submetidos a testes não invasivos, tornam-se opção viável para estudos das alterações da postura corporal. Sob o ponto de vista ortopédico, as avaliações posturais em escolares são benéficas e proporcionam oportunidade individual de diagnóstico precoce $^{7}$.

A fisioterapia ainda é pouco atuante no campo escolar, o que denota a necessidade de maior atenção, especialmente em relação aos aspectos preventivos posturais. Diante das evidências apresentadas, destaca-se a relevância científica e social de se investigar os principais desvios posturais da coluna vertebral, dentre eles a escoliose em adolescentes.

A realização deste estudo dá-se pela necessidade de se aprofundar o conhecimento dos agravos e eventos relacionados com a saúde do adolescente, em especial os desvios posturais da coluna vertebral $^{8}$.

\section{METODOLOGIA}

Foi realizado um estudo do tipo transversal e analítico. A amostra foi selecionada a partir de um universo de 2.432 alunos entre 11 e 19 anos, que estavam matriculados e que frequentavam uma das 67 salas de aula do turno diurno das 3 escolas do Ensino Fundamental II e Médio do município de Juazeiro do Norte, no estado do Ceará9.

A amostra foi calculada usando a técnica de estratificação de acordo com o nível de escolaridade. Foram sorteados aleatoriamente, conforme presença em sala de aula, 10 alunos (distribuídos equitativamente entre o sexo masculino e o sexo feminino) de cada sala, perfazendo uma amostra total de 670 alunos.

Foram excluídos os estudantes do turno noturno, por tratar-se, nesse período, de área com risco de segurança, bem como alunos portadores de doenças, uma vez que o objetivo desse estudo foi observar alterações em alunos habitualmente sadios.

Com o objetivo de facilitar a visualização, o exame físico foi realizado nos alunos com roupas de banho, sendo feitas demarcações de referência no corpo deles para facilitar a análise postural pela fotogrametria. Com a utilização de um simetrógrafo e de uma máquina fotográfica, pesquisou-se qualitativamente a presença de desvios na coluna vertebral por meio de duas fotografias por sujeito estudado (a presença de gibosidade na flexão anterior do tronco foi observada no plano frontal em vista posterior, e, em posição bípede, inspecionou-se a simetria de ombros e da pelve bem como os desvios laterais da coluna vertebral).

Foram usados nível d'água e régua para aferir a gibosidade dorsal e/ou lombar, além de um fio de prumo e de uma régua para medir os desvios laterais da coluna vertebral. Também utilizou-se um divã tubular para acomodar o aluno ao ser examinado em decúbito dorsal e uma fita métrica para medir o comprimento dos membros inferiores, da espinha ilíaca ântero-superior (EIAS) ao maléolo medial ipsilateral.

Por fim, o diagnóstico do possível desvio encontrado foi baseado no exame físico, sendo considerado como ponto de corte as assimetrias posturais, com base no alinhamento global dos segmentos do corpo encontrado nos planos acima descritos; as assimetrias de membros inferiores (MMII), considerando o encurtamento quando maior que $1 \mathrm{~cm}$; a escoliometria a nível dorsal com medida superior a 0,8 cm e a nível lombar acima de $0,5 \mathrm{~cm}$, caracterizando a presença de gibosidade dorsal e/ou lombar e, por conseguinte, a possível escoliose $\mathrm{e}^{10,11}$.

O registro fotográfico e a avaliação postural foram realizados pelo pesquisador, sendo entregue um laudo do exame a todos os alunos submetidos a ele, bem como uma carta de encaminhamento ao serviço público de saúde para possível realização de radiografia panorâmica de coluna toracolombar em antero-posterior (AP) àqueles que apresentaram os referidos desvios.

Para efeito do cruzamento de dados, foram consideradas como variáveis categóricas: sexo; hábitos laborais (trabalha ou não); hábitos escolares (uso de mochila, mão que escreve e tipo de cadeira); assimetrias de ombros ilíacos ou MMII; desvios laterais da coluna vertebral; presença de gibosidade e história familiar de deformidade postural. As variáveis quantitativas foram: idade; peso; altura e índice de massa corporal (IMC). Os dados gerais foram analisados 
de forma descritiva utilizando as frequências absolutas e percentuais. $\mathrm{Na}$ análise inferencial, foram utilizados os testes $\chi^{2} \mathrm{e}$ Exato de Fisher ao nível de significância de $5 \%(p<0,05)$. O tratamento estatístico foi realizado pelo programa EPI - Info 6.04 .

O estudo foi submetido e aprovado pelo Comitê de Ética em Pesquisa. Os alunos selecionados e que aceitaram participar do estudo assinaram um Termo de Consentimento Livre e Esclarecido de acordo com o preconizado pela resolução 196/96.

\section{RESULTADOS}

De acordo com a Tabela 1, pode-se observar que a prevalência de alterações posturais nos estudantes foi de $6,9 \%, 7,8 \%$ e $8,8 \%$ para as assimetrias de ombro, ilíaco e desvio lateral da coluna, respectivamente. Dentre os tipos de desvio da coluna, os principais tipos presentes foram: dorso-lombar à direita; dorsal à direita; lombar à direita e lombar à esquerda. Verificou-se também que a escoliometria para a coluna dorsal e a escoliometria para a coluna lombar estiveram presentes. No entanto, a gibosidade esteve presente em 16 $(2,4 \%)$ estudantes, sendo que 50,0\% deles apresentaram gibosidade do tipo dorsal à direita. Em relação à assimetria de MMII, apenas 1,0\% dos estudantes apresentaram tal deformidade.

Ainda na Tabela 1, observa-se que os estudantes relataram a existência de alguma deformidade postural na família, classificadas como escoliose, cifose, lordose ou mista, sendo a cifose e a escoliose as mais frequentes.

Em relação ao sexo, observa-se na Tabela 2 que a escoliose ocorreu com maior frequência no sexo feminino $(1,34 \%)$, sendo a maior proporção de casos ocorridos na faixa etária menor que 17 anos.

Conforme a Tabela 2, não foi encontrada associação significativa entre escoliose e as seguintes variáveis estudadas: sexo, idade, peso, altura, IMC $<20 \mathrm{~kg} / \mathrm{m}^{2}$, trabalho, uso de mochila, mão que escreve e tipo de cadeira. No entanto, foi encontrada associação significativa entre escoliose e assimetrias de ombros e ilíacos.
Tabela 1. Prevalência de alterações posturais e história familiar de deformidade postural

\begin{tabular}{|c|c|c|}
\hline Variáveis & $\mathrm{n}$ & $\%$ \\
\hline \multicolumn{3}{|l|}{ Assimetria de ombros } \\
\hline Presente & 46 & 6,90 \\
\hline Ausente & 624 & 93,10 \\
\hline \multicolumn{3}{|l|}{ Assimetria de ilíacos } \\
\hline Presente & 52 & 7,80 \\
\hline Ausente & 618 & 92,20 \\
\hline \multicolumn{3}{|l|}{ Desvio lateral de coluna } \\
\hline Presente & 59 & 8,80 \\
\hline Ausente & 611 & 91,20 \\
\hline \multicolumn{3}{|l|}{ Principais tipos de desvio presente } \\
\hline Dorsal à direita & 8 & 13,56 \\
\hline Dorsal à esquerda & 2 & 3,39 \\
\hline Lombar à direita & 7 & 11,86 \\
\hline Lombar à esquerda & 7 & 11,86 \\
\hline Dorso-lombar à direita & 17 & 28,82 \\
\hline Dorso-lombar à esquerda & 6 & 10,17 \\
\hline Dorsal à direita e lombar à esquerda & 7 & 11,86 \\
\hline Dorsal à esquerda e lombar à direita & 5 & 8,48 \\
\hline \multicolumn{3}{|l|}{ Escoliometria $>0,8 \mathrm{~cm}$ para coluna dorsal } \\
\hline Presente & 12 & 1,80 \\
\hline Ausente & 658 & 98,20 \\
\hline \multicolumn{3}{|l|}{ Escoliometria $>0,5 \mathrm{~cm}$ para coluna lombar } \\
\hline Presente & 7 & 1,00 \\
\hline Ausente & 663 & 99,00 \\
\hline \multicolumn{3}{|l|}{ Gibosidade } \\
\hline Presente & 16 & 2,40 \\
\hline Ausente & 654 & 97,60 \\
\hline \multicolumn{3}{|l|}{ Principais tipos de gibosidade } \\
\hline Dorsal à direita & 8 & 50,00 \\
\hline Dorsal à esquerda & 1 & 6,25 \\
\hline Lombar à direita & 2 & 12,50 \\
\hline Lombar à esquerda & 2 & 12,50 \\
\hline Dorso-lombar à direita & 1 & 6,25 \\
\hline Dorsal à direita e lombar à esquerda & 2 & 12,50 \\
\hline \multicolumn{3}{|l|}{ Assimetria de $M M I I>1 \mathrm{~cm}$} \\
\hline Presente & 7 & 1,00 \\
\hline Ausente & 663 & 99,00 \\
\hline \multicolumn{3}{|l|}{ História familiar de deformidade postural } \\
\hline Presente & 168 & 25,10 \\
\hline Ausente & 502 & 74,90 \\
\hline
\end{tabular}


Tabela 2. Frequência de escoliose segundo as variáveis, demográficas e antropométricas, e hábitos em adolescentes escolares

\begin{tabular}{|c|c|c|c|c|c|}
\hline \multirow{3}{*}{ Variável } & \multicolumn{5}{|c|}{ Frequência de escoliose } \\
\hline & \multicolumn{2}{|c|}{ Sim } & \multicolumn{2}{|c|}{ Não } & \multirow{2}{*}{ Valor $\mathrm{p}$} \\
\hline & $\mathrm{n}$ & $\%$ & $\mathrm{n}$ & $\%$ & \\
\hline \multicolumn{6}{|l|}{ Sexo } \\
\hline Masculino & 7 & 1,04 & 328 & 48,96 & \\
\hline Feminino & 9 & 1,34 & 326 & 48,66 & $0,613^{* *}$ \\
\hline \multicolumn{6}{|l|}{ Idade } \\
\hline 11 a 13 anos & 6 & 0,90 & 264 & 39,40 & \\
\hline 14 a 16 anos & 7 & 1,04 & 311 & 46,42 & \\
\hline 17 a 19 anos & 3 & 0,45 & 79 & 11,79 & $0,717^{* *}$ \\
\hline \multicolumn{6}{|l|}{ Peso (kg) } \\
\hline$<40$ & 4 & 0,60 & 158 & 23,58 & \\
\hline 40 a 50 & 7 & 1,04 & 249 & 37,16 & \\
\hline$>50$ & 5 & 0,75 & 247 & 36,87 & $0,703^{*}$ \\
\hline \multicolumn{6}{|l|}{ Altura (m) } \\
\hline$<1,50$ & 1 & 0,15 & 168 & 25,07 & \\
\hline 1,50 a 1,54 & 5 & 0,75 & 145 & 21,64 & \\
\hline 1,55 a 1,59 & 6 & 0,90 & 141 & 21,04 & \\
\hline$\geq 1,60$ & 4 & 0,60 & 200 & 29,85 & $0,261^{*}$ \\
\hline \multicolumn{6}{|l|}{ IMC (kg/m²) } \\
\hline$\leq 20$ & 14 & 2,09 & 399 & 59,55 & \\
\hline$>20$ & 2 & 0,30 & 255 & 38,06 & $0,191^{* *}$ \\
\hline \multicolumn{6}{|l|}{ Trabalha } \\
\hline Sim & 3 & 0,45 & 76 & 11,34 & \\
\hline Não & 13 & 1,94 & 578 & 86,27 & $0,421^{*}$ \\
\hline \multicolumn{6}{|l|}{ Uso de mochila } \\
\hline Sim & 1 & 0,15 & 75 & 11,19 & \\
\hline Não & 15 & 2,24 & 579 & 86,42 & $1,000^{*}$ \\
\hline \multicolumn{6}{|l|}{ Mão que escreve*** } \\
\hline Direita & 14 & 2,09 & 613 & 91,77 & \\
\hline Esquerda & 1 & 0,15 & 40 & 5,99 & $1,000^{*}$ \\
\hline \multicolumn{6}{|l|}{ Tipo de cadeira**** } \\
\hline Individual com braço à direita & 13 & 1,97 & 541 & 81,72 & \\
\hline Cadeira dupla & 3 & 0,45 & 105 & 15,86 & $0,734^{*}$ \\
\hline \multicolumn{6}{|l|}{ Assimetria de ombros } \\
\hline Presente & 8 & 1,19 & 38 & 5,68 & \\
\hline Ausente & 8 & 1,19 & 616 & 91,94 & $0,000^{* *}$ \\
\hline \multicolumn{6}{|l|}{ Assimetria de ilíacos } \\
\hline Presente & 15 & 2,24 & 37 & 5,52 & \\
\hline Ausente & 1 & 0,15 & 617 & 92,09 & $0,000 * *$ \\
\hline
\end{tabular}

*Teste Exato de Fisher; ${ }^{* *}$ Teste $\chi^{2} ;{ }^{* * *}$ Dois adolescentes que escreviam com ambas as mãos foram excluídos; ${ }^{* * * *}$ Oito adolescentes que usavam cadeira individual com braço lateral à esquerda foram excluídos
DISCUSSÃO

Objetivando investigar os principais desvios posturais da coluna vertebral em escolares adolescentes, o presente estudo encontrou elevada prevalência de escoliose. Segundo Perdriolle ${ }^{12}$, a frequência de escoliose é relatada por diversos autores entre 30 e $80 \%$, sendo $40 \%$ o índice mais frequentemente citado. Atualmente, os especialistas convergem em direção à hereditariedade multifatorial, podendo estar associada ao retardo de maturação do equilíbrio corporal, relacionado, por sua vez, ao sistema músculo-esquelético e a problemas metabólicos. Segundo Coillard e Rivard ${ }^{13}$, 80\% das escolioses são consideradas de origem idiopática, e diversas hipóteses buscam explicar o seu surgimento, dentre elas: fatores genéticos; esqueléticos; miogênicos; neurogênicos; assim como associação de diferentes fatores.

Os dados relativos à presença de desvios laterais da coluna vertebral encontrados nesta pesquisa são superiores à prevalência encontrada em estudos anteriores com população geral, que variou de 1 a $3 \%{ }^{14}$. Andersen et al. ${ }^{15}$ confirmaram que a prevalência global da escoliose encontra-se entre 1 e $2 \%$, e segundo esse estudo os adolescentes são o subgrupo mais comumente afetado pela escoliose idiopática. A prevalência de escoliose em escolares entre 10 e 14 anos de idade, de ambos os sexos, em estudo dirigido por Nery et al. ${ }^{16}$ foi de $1,4 \%$, assemelhando-se a encontrada por Rocha et al. ${ }^{17}$. É possível que a diferença encontrada em relação à prevalência de desvios laterais da coluna vertebral seja devido aos diferentes métodos nos estudos, apesar de estes terem analisado faixas etárias semelhantes.

A superioridade encontrada em relação à presença de desvios laterais no presente estudo, quando comparado com os resultados de outros estudos ${ }^{14,15}$, mostra uma realidade preocupante no contexto estudado, visto que as alterações posturais modificam os pontos de pressão sobre os segmentos corporais e podem causar problemas como dores nas costas, senão no momento atual, provavelmente em uma idade mais avançada ${ }^{18}$.

Um aspecto relevante foi o fato de ter sido encontrado o desvio dorso-lombar 
à direita como o tipo predominante de desvio neste estudo, o que se contrapõe aos dados referidos por Kisner e Colby ${ }^{19}$, que afirmam que a escoliose geralmente envolve as regiões torácica e lombar, podendo apresentar-se em " $\mathrm{S}$ " tipicamente em destros com curvatura para direita na região torácica e esquerda na lombar, ou leve curvatura em " $C$ " para esquerda nas regiões torácica e lombar. Porém, pode ainda haver assimetria nos ombros, pelve e membros inferiores, fato que está de acordo com as observações no presente estudo.

Em relação ao lado da convexidade da curva mais acometido, outros estudos divergem do resultado encontrado nesta pesquisa. Fornazari e Pereira ${ }^{20}$ apresentaram que 53\% das curvaturas na região torácica localizavam-se à esquerda. Em concordância, no estudo de Minghelli ${ }^{5}$ as curvas foram analisadas em relação ao seu número e ao lado da sua convexidade, além de ser verificada a predominância de desvios na região torácica, representando $17,3 \%$ dos desvios, sendo $7,9 \%$ à direita e $9,4 \%$ à esquerda.

Em relação ao sexo, os achados do presente estudo estão de acordo com os de Minghelli ${ }^{5}$, que também encontrou maior prevalência em meninas. $\mathrm{O}$ estudo realizado por Nery et al. ${ }^{16}$ mostrou prevalência de $1,98 \%$ entre as meninas e de $0,8 \%$ entre os meninos. Taylor e Twomey $^{21}$ relatam que esse desvio em indivíduos do sexo feminino tem maior probabilidade de progressão devido à forma do corpo vertebral e do menor suporte muscular. É possível que essas diferenças encontradas tenham ocorrido em virtude de o pico de crescimento do corpo das mulheres ocorrer mais cedo que nos rapazes, tornando assim mais fácil detectar a deformidade caso o estudo tenha sido realizado no período em que os rapazes ainda não tenham vivenciado o pico de crescimento $^{16}$.

Com relação à proporção dos casos de acordo com as faixas etárias, na pesquisa de Pereira et al. ${ }^{1}$ realizada com ambos os sexos, a frequência de casos suspeitos foi maior no grupo etário de 13 a 15 anos. No estudo de Minghelli ${ }^{5}$, os dados relativos à idade revelaram maior prevalência de posturas escolióticas na idade de 11 anos. Porém, os resultados desse estudo indicam que não há relação significativa entre a idade e as posturas escolióticas.

Esses dados evidenciam a relevância dos programas de detecção precoce dos desvios no período da adolescência. Segundo Ortiz ${ }^{22}$, as possíveis malformações vertebrais ocorrem antes do desenvolvimento dos centros de ossificação.

Não foi encontrada associação significativa entre escoliose e IMC $<20 \mathrm{~kg} / \mathrm{m}^{2}$, ou seja, em adolescentes com baixo peso. Tal fato contrapõe-se aos achados que tratam sobre carências nutricionais durante a fase de crescimento e desenvolvimento, referindo-se às carências como fator de risco para a escoliose. É o que ocorre no estudo de Damsim et al. ${ }^{23}$, relatando que inúmeros fatores interferem no processo evolutivo, alguns geneticamente programados, enquanto outros como mecânicos, biológicos e medicamentosos são capazes de modificar, alterar ou interferir no crescimento, podendo assim gerar desvios posturais.

Complementando os achados de Damsim et al. ${ }^{23}$ sobre desvios posturais e baixo IMC, Martelli e Traebert ${ }^{4}$ descrevem que menor estatura e menor peso corporal estão estatisticamente associados à ocorrência de hiperlordose relacionada à coluna cervical. Porém, no estudo de Detsch ${ }^{7}$, as alunas com IMC normal apresentaram maior prevalência de alterações na coluna vertebral se comparadas com aquelas com sobrepeso ou obesidade. Mesmo após a análise multivariada, os desvios posturais laterais continuaram associados com o $\mathrm{IMC}^{7}$.

Em oposição, Brandalize e Leite ${ }^{24}$ observaram que crianças e adolescentes obesos são mais predispostos a desenvolver complicações ortopédicas que os indivíduos eutróficos, sendo que, no estudo citado, os principais problemas relatados foram: alterações posturais, como hiperlordose lombar e joelhos valgos, e dores músculo-esqueléticas principalmente na coluna lombar e membros inferiores. Essas alterações posturais não foram constatadas na presente pesquisa.

Segundo Kendall et al. ${ }^{11}$, a assimetria de ombros pode estar relacionada ao lado dominante do sujeito. Raine e Twomey ${ }^{25}$ discutiram essa relação a partir do pressuposto que o ombro mais baixo poderia corresponder ao lado dominante.
Eles notaram em seus achados que o ombro direito é frequentemente mais baixo um grau que o esquerdo.

Mighelli ${ }^{5}$ relata achados referentes à presença de assimetria e à diferença entre o comprimento dos membros inferiores em indivíduos com posturas escolióticas. Diferentemente do estudo de Mighelli ${ }^{5}$, em nosso estudo não foi encontrada uma relação significativa entre variáveis assimetria e postura escoliótica.

A relação da assimetria com a escoliose ainda não está bem estabelecida, sendo que esta pode ser o fator desencadeante da curvatura ou apenas uma consequência. Chagas et al. ${ }^{26}$ consideram que os músculos efetores do movimento são, em situações de desequilíbrio, produtores de deformidades esqueléticas.

Howard et al. ${ }^{27}$ alertam que grandes curvas sem tratamento progridem substancialmente durante a vida adulta e causam aumento da deformidade, podendo reduzir a expectativa de vida, além de possuir grande impacto na vida diária como dor lombar.

Cabe ressaltar que o presente estudo teve as seguintes limitações: não foram analisados sinais e sintomas específicos da dor como localização, intensidade e irradiação; não possibilitou o estabelecimento de uma relação temporal de causa e efeito devido ao tipo de delineamento utilizado.

Portanto, recomendamos a realização de intervenções, que tenham como finalidade desenvolver e avaliar a aplicação de ações educativas por meio de atividade física, e de intervenções fisioterapêuticas, com o objetivo de prevenir as algias de coluna vertebral em adolescentes. Segundo Zapater et al. ${ }^{28}$, a fisioterapia preventiva é capaz de incluir programas de educação postural que possibilitam o conhecimento acerca dos desvios posturais, minimizando as complicações entre os escolares.

\section{CONCLUSÃO}

Foi encontrada maior predominância de escoliose em portadores de assimetrias de ombros e em portadores de assimetrias de ilíacos, além da predominância de escoliose no sexo feminino. Porém, não foi constatada influência de peso, altura e IMC para a prevalência de escoliose. 


\section{REFERÊNCIAS}

1. Pereira LM, Barros PCC, Oliveira MND, Barbosa AR. Escoliose: triagem em escolares de 10 a 15 anos. Rev Saúde Com. 2005;1(2):134-43.

2. Iunes DH, Cecílio MBB. Análise quantitativa do tratamento da escoliose idiopática com o método klapp por meio da biofotogrametria computadorizada. Rev bras fisioter. 2010;14(2):133-40.

3. Alexandre NMC, de Moraes MAA. Modelo de avaliação físico-funcional da coluna vertebral. Rev Lat Am Enfermagem. 2001;9(2):67-75.

4. Martelli RC, Traebert J. Estudo descritivo das alterações posturais de coluna vertebral em escolares de 10 a 16 anos de idade, Tangará-SC, 2004. Rev bras epidemiol. 2006;9(1):87-93.

5. Minghelli B. Rastreio escolar: a importância na detecção precoce de posturas escolióticas em adolescentes das escolas de Silves, Algarve. Rev Port Sal Pub. 2008;26(2):61-8.

6. Cabral LTB, Filho ESV, Ueno FH, Yonezaki AM, Rodrigues LMR. Avaliação da qualidade de vida em pacientes com escoliose idiopática do adolescente após tratamento cirúrgico pelo questionário SF-36. Coluna/Columna. 2009;8(3):315-22.

7. Detsch C, Luz AMH, Candotti CT, de Oliveira DS, Lazaron F, Guimarães LK, et al. Prevalência de alterações posturais em escolares do ensino médio em uma cidade no Sul do Brasil. Rev Panam Salud Publica. 2007;21(4):231-8.

8. Braccialli LMP, Vilarta R. Aspectos a serem considerados na elaboração de programas de prevenção e orientação de problemas posturais. Rev paul educ fís. 2000;14(2):159-71.

9. Inep. [Internet]. Censo Escolar. [citado 2005 out 10]. Disponível em: http:///www.inep.gov.br.

10. Bremberg S, Nilsson-Berggren B. School screening for adolescent idiopathic scoliosis. J Pediatr Orthop. 1986;6(5):564-7.

11. Kendall FP, Mccreary EK, Provance PG. Músculos: provas e funções. 4. ed. São Paulo: Manole; 1995.

12. Perdriolle R. Mechanical process and growth cartilages. Essential factors in the progression of scoliosis. Spine. 1993;18(3):343-9.

13. Coillard C, Rivard CH. Vertebral deformities and scoliosis. Eur Spine J. 1996;5(1):91-100.

14. Brooks HL, Azen SP, Gerberg E, Brooks R, Chan L. Scoliosis: A prospective epidemiological study. J Bone Joint Surg Am. 1975;57(7):968-72.
15. Andersen MO, Thomsen K, Kyvik KO. Adolescent idiopathic scoliosis in twins: a population-based survey. Spine. 2007;32(8):927-30.

16. Nery LS, Halpern R, Nery PC, Nehme KP, Stein AT. Prevalence of scoliosis among school students in a town in southern Brazil. São Paulo Med. J. 2010;128(2):69-73.

17. Rocha LE, Santili C, Carrera EF, et al. Escolioses idiopáticas. In: Sociedade Brasileira de Ortopedia e Traumatologia. Ortopedia pediátrica. Rio de Janeiro: Revinter, 2004:115-30.

18. Iwamoto J, Abe H, Tsukimura Y, Wakano K. Relationship between radiographic abnormalities of lumbar spine and incidence of low back pain in high school rugby players: a prospective study. Scand J Med Sci Sports. 2005;15(3):163-8.

19. Kisner C, Colby LA. Exercícios terapêuticos: fundamentos e técnicas. 3. ed. São Paulo: Manole; 1998.

20. Fornazari LP, Pereira VCG. Prevalência de postura escoliótica em escolares do ensino fundamental. Cad. Escola de Saúde Fisioterapia. 2008;1(1):1-13.

21. Taylor JR, Twomey LT. The role on the notochord and blood vessels in vertebral column development and in the aetiology os Schmorl's nodes. In Manual therapy, 1, The vertebral column. Edinburgh: Churchill Livingsotone; 1984.

22. Ortiz J. Coluna toracolombar: deformidades. In Hebert S, Xavier R. Ortopedia e traumatologia: princípios e prática. São Paulo: Artmed; 2003:111-49.

23. Damsin JP, Djenadi K, Josset P, Wioland M. Cartilage de croissance et croissance en orthopédie. Encycl Med Chir (Elsevier, Paris), Appareil locomoteur. 1996;14(009A)10:14.

24. Brandalize $M$, Leite N. Alterações ortopédicas em crianças e adolescentes obesos. Fisioter mov. 2010;23(2):283-8.

25. Raine S, Twomey LT. Head and shoulder posture variations in 160 asymptomatic women and men. Arch Phys Med Rehabil. 1997;78(11):1215-23.

26. Chagas JCM, Schimidt B, Puerta EB, Oliveira CEAS, de Freitas AA. Estudo histoquímico dos músculos rotadores do dorso em pacientes com escoliose idiopática do adolescente. Rev bras ortop. 1998;33(2):111-8.

27. Howard A, Donaldson S, Hedden D, Stephens D, Alman B, Wright J. Improvement in quality of life following surgery for adolescent idiopathic scoliosis. Spine. 2007;32(24):2715-8.

28. Zapater AR, Silveira DM, de Vitta A, Padovani CR, da Silva JCP. Postura sentada: a eficácia de um programa de educação para escolares. Ciênc saúde coletiva. 2004;9(1):191-9. 